\title{
A framework for communicating the utility of models when facing tough decisions in public health
}

\author{
Jason Thompson ${ }^{1,2}$, Rod McClure ${ }^{3}$, Nick Scott ${ }^{4,5}$, Margaret Hellard ${ }^{4}$, Romesh Abeysuriya ${ }^{4,5}$, Rajith \\ Vidanaarachchi ${ }^{1,2}$, John Thwaites ${ }^{5}$, Jeffrey V. Lazarus ${ }^{6}$, Susan Michie ${ }^{7}$, Chris Bullen ${ }^{8}$, Mikhail Prokopenko ${ }^{9}$ \\ ${ }^{12}$, Sheryl L. Chang ${ }^{9}$, Oliver M. Cliff ${ }^{9}{ }^{10}$, Cameron Zachreson ${ }^{9,11}$, Tony Blakely ${ }^{13}$, Tim Wilson ${ }^{13}$, Driss Ait \\ Oakrim $^{13}$, Vijay Sundararajan ${ }^{13,14}$, Kelly $\mathrm{O}^{\prime}$ Shea $^{15}$, Sarah Bartsch ${ }^{15}$, Bruce Lee $^{15}$ \\ ${ }^{1}$ Transport, Health and Urban Designed (THUD) Research Laboratory, Melbourne School of Design, The \\ University of Melbourne, Australia \\ ${ }^{2}$ Centre for Human Factors and Sociotechnical Systems, The University of the Sunshine Coast, Australia \\ ${ }^{3}$ Faculty of Medicine and Health, University of New England, Australia \\ ${ }^{4}$ Burnet Institute, Melbourne, Australia \\ ${ }^{5}$ Monash University, Melbourne, Australia \\ ${ }^{6}$ Barcelona Institute for Global Health (ISGlobal), Hospital Clinic, University of Barcelona, Spain \\ ${ }^{7}$ Centre for Behaviour Change, University College London, United Kingdom \\ ${ }^{8}$ National Institute for Health Innovation, The University of Auckland, New Zealand \\ ${ }^{9}$ Centre for Complex Systems, The University of Sydney \\ ${ }^{10}$ School of Physics, The University of Sydney, Australia \\ ${ }^{11}$ School of Computing and Information Systems, The University of Melbourne, Australia \\ ${ }^{12}$ Sydney Institute for Infectious Diseases, The University of Sydney, Australia \\ ${ }^{13}$ School of Population and Global Health, The University of Melbourne, Australia \\ ${ }^{14}$ Department of Public Health, La Trobe University, Melbourne, Australia \\ ${ }^{15}$ Center for Advanced Technology and Communication in Health (CATCH), \\ City University of New York
}

The COVID-19 pandemic has brought the discipline of public health, infectious disease, and policy modeling squarely into the spotlight. Never before have decisions regarding public health measures and their impacts been such a topic of international deliberation from the level of individuals and communities through to global leaders. And nor previously have models - developed at rapid pace and often in the absence of complete information - been so central to the decision-making process. However, after more than 18 months of experience with pandemic modeling, policy-makers need to be more confident about which models will be most helpful to support them when taking public health decisions. We combine the authors' collective international experience of modelling for and with Governments and policy-makers with prior research utilisation scholarship to describe a framework to assist both modelers and policy-makers consider the utility of models that may be available to them when faced with difficult public health and policy decisions. To illustrate these principles, a set of three independent but complementary modeling case-studies undertaken at the same time in NSW, Australia during that state's unfolding second wave of COVID-19 infections is presented. 

Standing side-by side with scientists and public health officials, leaders and policy-makers announcing difficult public health measures and social restrictions in response to surging COVID-19 cases, vaccine sideeffects, thresholds, or business and social pressures have been at pains to explain that they are 'following the science' and/or the 'advice of health and medical experts'.

The vison of science and medicine embodied here is of its popular conceptualisation; white coats, laboratories, stethoscopes, test-tubes, highly controlled experiments, and observation. Yet in reality, the science driving much of the decision-making likely looks very different. The modelling aspects of the scientific effort can resemble a dispersed network of computational modelers and mathematicians drawing upon multiple sources of data and expertise from across the public and academic realm, deploying software models on remote, high performance computing clusters. These efforts utilise information from several quarters (e.g., laboratory science, epidemiological evidence, and behavioural science) and then consolidate and integrate these data alongside expert judgement and abstractions of social norms, dynamics, networks and structures, interactions, cognition and patterns of movement to generate an overall picture of the world, its mechanics and trajectory.

These representations are often agent-based models $(A B M s)^{1}$ that mirror fine-grained artificial societies, while others are system dynamics models, compartmental models, discrete event simulation models, and other associated mathematical representations. Each approach has unique strengths, weaknesses, and levels of sophistication and complexity ${ }^{2}$.

Computational models can provide effective tools for exploring complex, dynamic relationships between multiple factors that affect the operation of systems by combining spatial, social, behavioural and biological phenomena in single representations ${ }^{3}$. When created as faithful representations of real social systems, computational models are useful for policy-makers because they afford an examination of policy scenarios and the direct and indirect effect of designed interventions within synthetic societies that can resemble 'virtual laboratories'. Public policy scenarios can be planned, run, and re-run, in a low-risk environment and prior to taking place in the real-world ${ }^{4}$.

However, despite their great potential, some computational models (such as ABMs) have been considered as 'black boxes' when compared with more standard mathematical models (e.g., compartmental models) that are not developed from a systems-thinking perspective. As a relatively new methodological paradigm $^{5}$, they can therefore fail to engage the trust of intended recipients ${ }^{6,7}$. This failure needs to be overcome if computational models are to reach their potential in assisting leaders to make better, more informed decisions.

\section{The purpose of using models in public health}

The purpose of using models in public health (e.g., COVID-19 responses) can be as a tool for structured decision making as well as informing, analysing, explaining, speculating, and planning ${ }^{8}$. Models enable decision makers to consider the impact of different variables (e.g., vaccination rates) and policy decisions (e.g., mask mandates, lockdowns) on population health outcomes ${ }^{9}$. They can also be used to estimate the health and cost effectiveness of different public health interventions ${ }^{10}$ or to assist policy-makers to navigate complexity by identifying downstream secondary or tertiary effects of policies. Beyond prediction, models can identify and explain emerging trends, key relationships and drivers of situations that are unfolding, thresholds at which events might occur (e.g., system collapse) ${ }^{11}$, or point us to missing data that will be important to collect in the future. 
The use of computational models to support public health decision-making is, however, nothing new. Multiple reports, articles and guidelines exist that lay out clear, methodical means of model development with policy-makers that include principles of collaboration, participation and iteration e.8., 12,13-16. However, most of these frameworks have been created under assumptions that both the modeler and user are not facing immediate crises that preclude lengthy development and collaboration cycles (e.g., a global infectious disease pandemic). Crises demand a different approach unlikely to follow a normal linear scientific pathway from information generation to utilisation ${ }^{17}$.

Policy-makers facing novel, urgent crises with deep uncertainty and limited historical record ${ }^{18,19}$ combined $^{2}$ with often restricted scientific understanding or training ${ }^{18,20-22}$ cannot politically nor ethically wait for the passing of 'normal' scientific processes and maturation of evidence to before acting ${ }^{4,23}$. When engaged with the science ${ }^{24,25}$, policy-makers quickly realise that formal models constructed amid crises need to incorporate available and/or sufficient evidence as a priority more-so than certainty ${ }^{14}$. This point was emphasised in the early stages of the SARS-CoV-2 pandemic by the Director of WHO's Health Emergencies Program, Mike Ryan who noted, "If you need to be right before you move, you will never win. Perfection is the enemy of the good when it comes to emergency management" ${ }^{\prime 23}$.

The key consideration for policy-makers facing problems or crises is therefore to know and recognise (i) features of models that indicate they will be useful at the point of the crisis they find themselves, and (ii) that the evidence generated by them is both timely and robust enough to be acted upon.

Drawing from research utilisation scholarship in the social sciences ${ }^{17,26}$, we propose adoption of a framework for policy-makers and advisors that affords a rapid and objective appraisal of models made available to them when faced with problems they must solve. In doing this, we recommend they consider three elements of model utility described below.

1) Instrumental utility, taking into account model:

a. Inputs

b. Mechanisms, and

c. Outputs

2) Conceptual utility, and

3) Political utility

\section{Model features that public health policy-makers should look for in times of crisis}

Instrumental utility requires the model to produce evidence that adequately answer questions posed of it by policy-makers. Ideally, policymakers would be involved in the design, development and framing of any model so they may answer questions about what interventions are possible, costs of interventions, and considerations that need to be included. Policy-makers should feel as though they are 'in' the model and can 'drive' it by manipulating its policy levers and experimenting with imagined scenarios. The model should look at a problem and attempt to solve it from the policy-maker's perspective, utilising expert insights distilled from the underlying simulations/modelling.

Evidence that has instrumental utility can be used to adjust or inform policy decisions facing governments and administrators (i.e., the evidence purports to show what policy levers are available, how and when those policies could be enacted, and what the consequences might be); the typical technical goal of modeling teams. A model that has instrumental utility is calibrated, valid, robust, and provides guidance that is as clear and accurate as possible given the uncertain nature of the crisis. As far as possible, the 
model should meet formal criteria for quality of theory, realism, mechanics, and objectivity as set out by the discipline(s) that contributed to its structure. It should be transparent, reproducible and able to generate results within an adequately short, policy-relevant timeframes.

The technical robustness of the model's construction is just as valuable as its theoretical robustness since minor technical errors can propagate into more significant errors, resulting in incorrect advice that, in turn, may lead to non-optimal policy decisions. A model formally defined according to the criteria set out by the contributing discipline(s) should be implemented with formal testing and verification methods where possible ${ }^{27}$. Transparent model checking ${ }^{28}$ using formal methods is crucial in maintaining a model's instrumental utility. For example, formal validation and verification used in electronic voting systems helps maintain public trust and policy-maker support ${ }^{29}$.

To assess the model's instrumental utility, we propose the user should compare three elements of what they know of the real world with what they understand of the presented model world. They should ask what the concordance is between the real world and model 1 ) inputs (e.g., features and elements being included in the model), 2) mechanisms (i.e., the way the model works by combining model inputs), and 3) outputs (the range of outcomes generated by the model that describe its performance) (see Figure 1). If there is a high degree of concordance across all three areas, the model is more likely to be a faithful representation of reality and its outcomes more likely to be trustworthy. Taking these elements individually, it is self-evident that poor input data will result in poor output data even if a models' mechanics are sound. Poor mechanics will turn even good input data into poor output data or misrepresent the way (i.e., policies) good policy outcomes might be achieved ${ }^{13}$. And poorly specified or limited outcome data (e.g., ignoring or failing to measure important unintended effects of policy decisions) will not provide policy-makers with adequate insight into the required range of outcomes they need (e.g., health and economic considerations of policy choices).

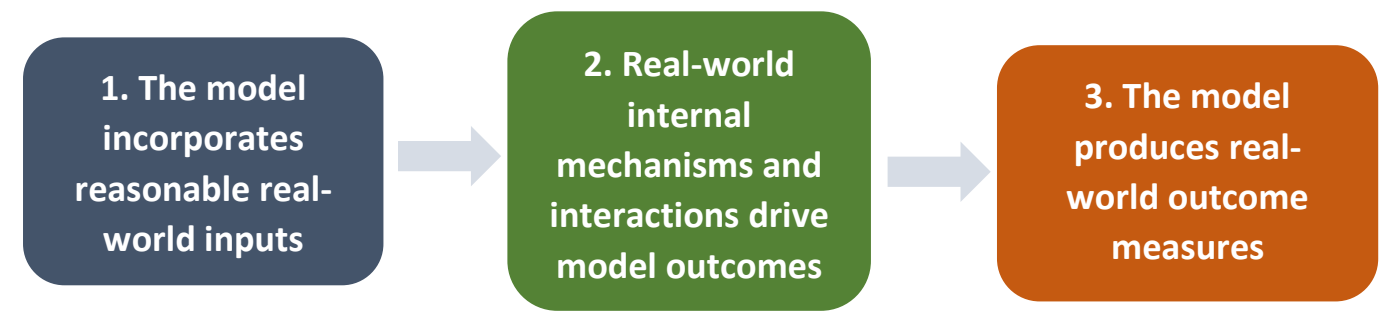

Figure 1. Qualities policy-makers should look for in models used to assist decision-making. 
Conceptual utility is the model's capacity to be effectively communicated, thereby convincing the public, policy-makers, advisors and co-decision-makers that the evidence produced by the model is robust, is explainable, makes sense, and can be trusted. Evidence with conceptual utility can influence policymakers, public servants, and general public perceptions around the need for policy action. In addition to instrumental utility, above, the model and the evidence it provides should also 'look and sound credible' to the public and those around the decision-making table. Model transparency as described above is important, as are the qualifications, expertise and backgrounds of the model producers. The team or individuals who created the model should be known, credible and respected by their peers. They should be perceived as independent, free from conflicts of interest, and / or from respected institutions. The model should broadly agree with evidence from other sources, but also generate occasional novel but explainable moments of insight for users. All in all, the model must deliver valuable insight that is not possible without it, and be seen to provide credible, transparent evidence that can be trusted and publicly defended.

For models to have political utility means their instrumental and conceptual utility is strong enough

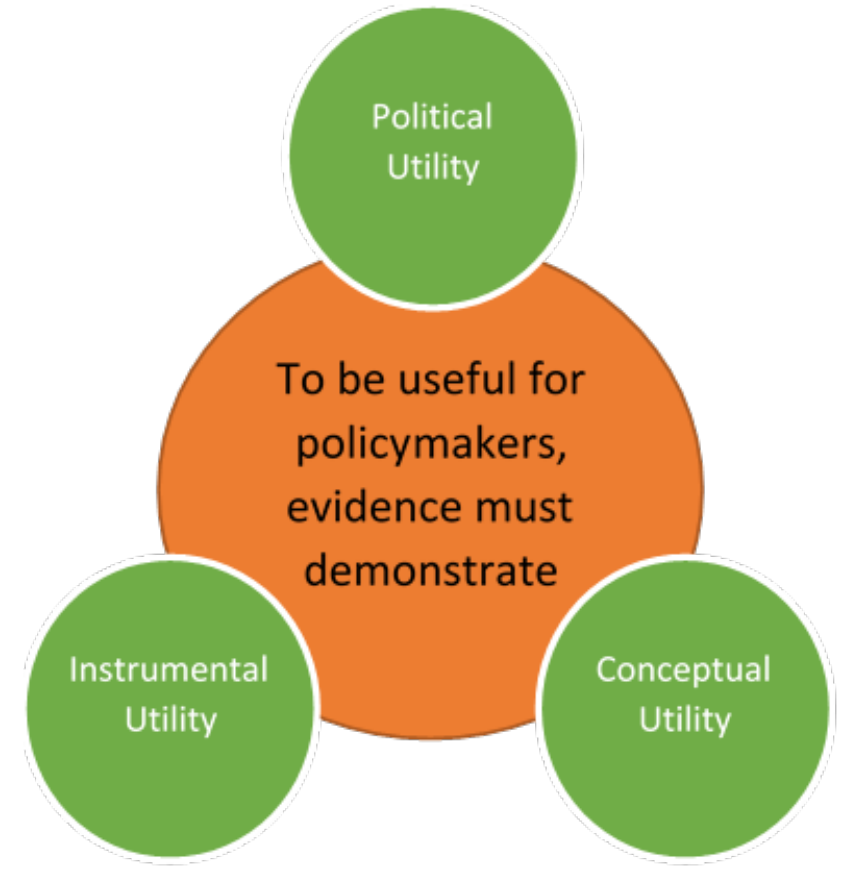

Figure 2. Three elements of instrumental, conceptual, and political utility that assist models be useful for policy-makers. to support defensible policy actions that will be supported by the community over extended time. Political utility enables the implications of the model to be actively and successfully woven into policy making and desired actions of Government. A model that does not have conceptual utility will not have political utility. A model that does not have instrumental utility will be found wanting through inaccuracy, so will also lose conceptual and eventually political utility. Once credibility is lost, trust in models will then be difficult to recover and future efforts may be met with increased scepticism, leading to reluctance on the part of the community to comply with policy-makers' directives.

Only when these three interdependent forms of utility derived from the model's outputs are satisfied are windows of opportunity ${ }^{30}$ likely to open for scientific evidence derived through models to successfully integrate with public policymaking.

Using these criteria, Table 1 provides a set of guidelines that policy-makers can use to evaluate whether any presented model approaches the thresholds for use in their local context. 
Table 1. Features of models that provide utility to policy-makers in times of crisis.

\begin{tabular}{|c|c|}
\hline UTILITY ELEMENT & MODEL CHARACTERISTICS \\
\hline $\begin{array}{l}\text { INSTRUMENTAL } \\
\text { UTILITY (THE } \\
\text { MODEL WORKS) }\end{array}$ & 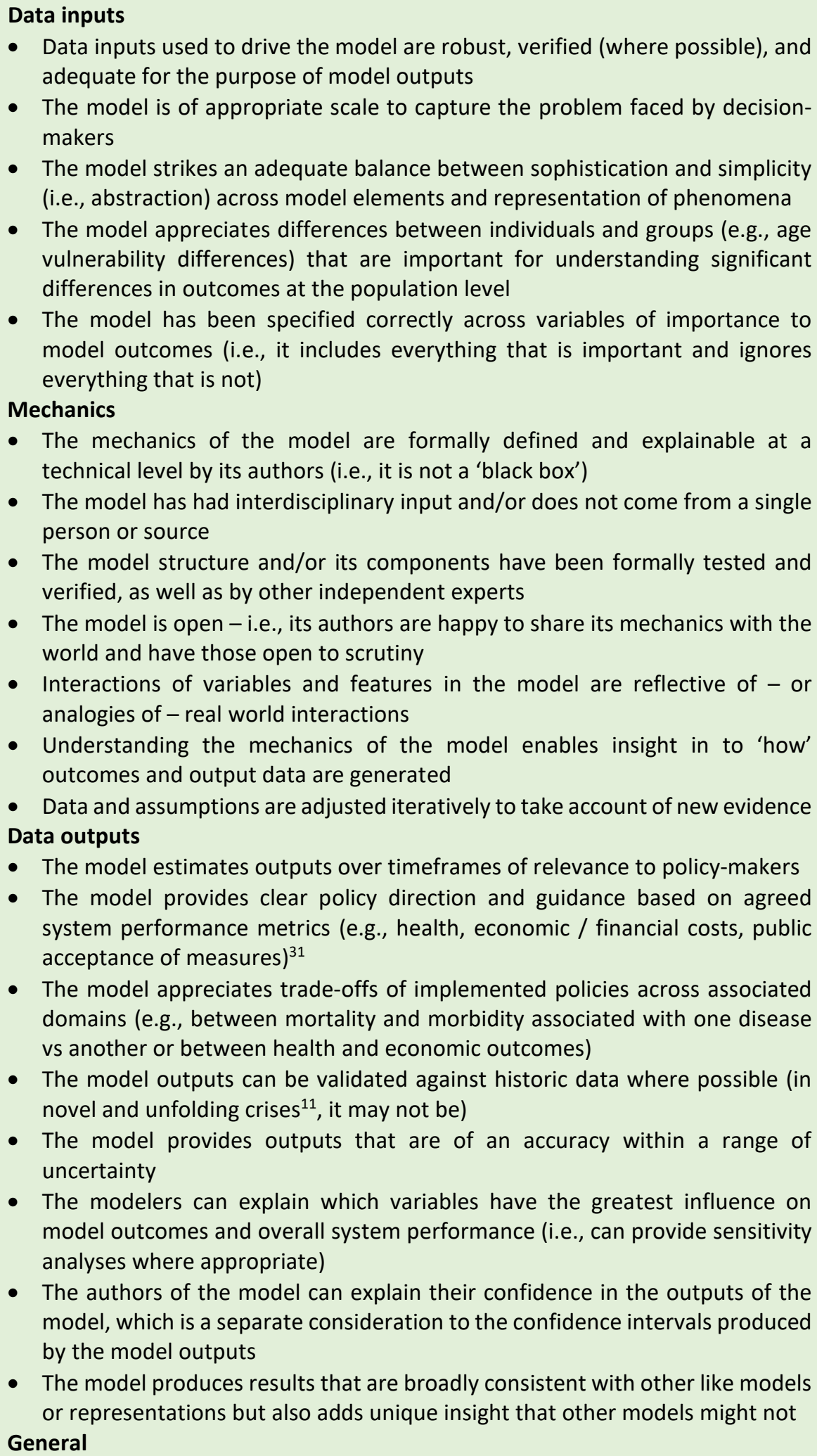 \\
\hline
\end{tabular}


CONCEPTUAL UTILITY (THE MODEL IS UNDERSTOOD)

POLITICAL UTILITY (THE POLICY IMPLICATIONS OF THE MODEL ARE SUPPORTED)
- The model framework, including assumptions and outcome measures, has been developed in collaboration with policy-makers (as far as possible)

- The model is fast enough to provide guidance in the timeframe required by policy-makers

- The model is of adequate scope to capture and reflect the problem faced by policy-makers

- The model is being used for the purposes it was designed

- The authors can clearly articulate what the model is missing, what level of detail it cannot capture, what it can't tell the user, and what it should not be used for

- The model is transparent - each aspect of it is explainable in plain language to a naïve audience - it is not a 'black box' that neither the model authors nor outside experts can explain

- The model looks and sounds credible to a naïve audience and/or the audience (e.g., general public) who will be subject to its recommendations

- The model authors and contributors are suitably qualified and experienced

- The model authors are independent and/or there is not apparent conflict of interest

- The model authors' institution is suitably qualified, experienced and their institution is independent from political decision-making

- The model appeals to common sense but is sophisticated enough to extend the boundaries of people's ability to conceptualise multiple future scenarios

- The model results are at times surprising but remain logical and explainable when surprising results emerge, demonstrating insight that might not otherwise have been gained through informal, implicit modeling

- Model results are presented plainly and implications are self-evident

- Model authors and/or their institution are presentable and can defend the validity of their work to the public

- The model has a public interface and/or can be manipulated by the public and/or other end-users to aid understanding

- The range of uncertainty in across model inputs and estimates is made clear to policy-makers

- The implications of the model can be woven into an acceptable, consistent political narrative by policy-makers

- The model has adequate instrumental utility as described above

- The model has adequate conceptual utility as described above

- Decision-makers (Ministers, public servants and health authorities) have input into the modeling and its assumptions.

- The model outputs and recommendations are accepted as robust by policymakers

- If policy-makers plan to use the model and/or its authors to prosecute public health initiatives, they are prepared to implement recommendations as per the model design and/or clearly articulate which aspects of the model they are taking recommendations from

- The model is / is proving to be accurate

- The model authors are / are proving to be reliable communicators and support its use in the way it is being used

- The relationship between model authors and policy-makers remains collaborative and productive 
- The public continue to support the policy implications of the model's findings

\section{Conclusions}

Computational policy modeling can be an extremely low cost but high value exercise for policy-makers faced with crises and critical decisions in public health. Despite this, computational models have not progressed far beyond the realm of 'toys' in the minds of some policy-makers ${ }^{6}$. Worse, poor models, poor explanations of model limitations, or poor experience with models and modeling teams can result in the field being dismissed as confusing, contradictory, and untrustworthy.

To help alleviate this problem, we have drawn upon our own experiences working with policy-makers during the COVID-19 pandemic as well as prior research utilisation scholarship to present a framework that asks both model developers and policy-makers to evaluate the utility of models across three dimensions. This framework demands more from researchers and model developers who intend that their work be used for decision-support in public health. We ask model developers to ensure their models work for the purposes they are intended, that they are transparent, comparable, well communicated. We ask that they demonstrate qualities of 1) instrumental, 2) conceptual, and 3) political utility.

Weiss ${ }^{17}$ laid out a challenging environment for researchers who desire to influence policy, and in the 4 decades since its publication, little has changed. Her insights demonstrate that modelers and modeling teams must be sophisticated in their approach, sensitive to issue of timing and politics, and committed to providing information to the right people, in usable form. She also suggests that to be influential, they might also be lucky.

"It probably takes an extraordinary concatenation of circumstances for research to influence policy decisions directly: a well-defined decision situation, a set of policy actors who have responsibility and jurisdiction for making the decision, an issue whose resolution depends on... information, identification of the requisite informational need, research that provides the information in the terms that match the circumstances within which choices will be made, research findings that are clear-cut, unambiguous, firmly supported, and powerful, that reach decisionmakers at the time they are wrestling with the issues, that are comprehensible and understood, and that do not run counter to strong political interests."

Computational modeling has the potential to act as a consolidating discipline that sits at the interface of science and public policy across the world. Greater integration and enhanced interaction between model developers and policy-makers on what creates a useful model is likely to improve the understanding and utilisation of models in public health, as well as the models, themselves. 


\section{Appendix 1}

\section{Case Studies}

The following three case studies are gathered from three independent research groups (The Burnet Institute, The University of Melbourne, and University of Sydney) that conducted modeling aimed at understanding the likely trajectory of COVID-19 cases in the Australian state of New South Wales during July, 2021, when that state was at the beginning of a significant outbreak. The case-studies are provided to enable direct comparison between modeling approaches and highlight differences, but to also demonstrate that ultimately, their implications for policy-makers differed only marginally.

The consistent message of all three models were that public health policy settings in place in NSW as of mid-July would be insufficient to drive infections lower in the short term.

The results of all each team's work were publicly released during July. At the time of writing (November $\left.6^{\text {th }}, 2021\right)$, the outbreak has been contained to around 200 new cases per day. 


\section{Case Study 1}

\begin{tabular}{l|l}
\hline Model & Burnet Institute, Institute for Disease Modelling, University of Copenhagen
\end{tabular}

Authors

Model

Architecture

Model

inputs and

starting

conditions

$$
\text { Covasim is an agent-based microsimulation model, developed in Python. }
$$

Agents in the model are assigned an age (which affects their susceptibility to infection and their disease prognoses), a household, and either a school (for people age 5-17) or a workplace (for people aged 18 to 65). Agents can also participate in multiple community activities including catching public transport, attending restaurants, pubs, places of worship, community sport, and small social gatherings. Agents are allocated setting-specific numbers of contacts associated with these activities, drawn from Poisson or Negative Binomial distributions. Transmission probabilities per contact, contact network structures and contact tracing capability vary by setting. For example, classrooms contact networks are completely connected clusters, involving the same agents each day, with contacts easily traced, whereas public transport contact networks involve regeneration of random edges between agents each day, and contacts less likely to be traced.

The model includes testing, contact tracing and quarantine of close contacts and their household contacts, isolation of confirmed cases, masks, physical distancing policies in venues (e.g., density limits), and policy restrictions to prevent or reduce transmission in different settings (e.g., closing schools, workplaces, or venues). It also includes vaccination with separate levels of protection against infection, symptoms, hospitalization, ICU and death.

Individuals are modelled to respond differently to COVID-19; whether they get tested, how long they wait before being tested, and compliance with quarantine are probabilistic. For people who become infected, age-specific probability distributions are used to determine time-varying viral load (which impacts onward transmission) and disease prognoses.

For this analysis we used the calibrated Victorian model, with a population of 100,000 agents and a single undiagnosed delta variant case seeded at random in the community. After the first diagnosis is recorded, a "density limits only" restriction policy is enacted. Multiple simulations were run starting from different initial seeds, and then filtered to retain only those that were within $20 \%$ of a 7-day average of 30 diagnoses per day, 21 days after the first case was detected, approximating the state of the epidemic in Sydney at the time of analysis.

Three scenarios were applied to the retained simulations: maintaining density limits, or escalation to the 'Stage 3' and 'Stage 4' restriction levels used in the Victoria in June-August 2020. The aim was to assess whether these restriction levels, combined with some vaccine coverage and improvements to contact tracing that have since occurred, would be sufficient to control an outbreak of the more infectious delta strain once it had reached a level comparable to the Sydney outbreak at the time of analysis. These restriction packages were chosen because their effect sizes were known from our previous model calibration to the Victorian second wave in 2020.

Results The model scenarios showed:

- With only density limits and masks, cases would continue to grow exponentially. 
- Stage 3 restrictions are sufficient to arrest growth of the outbreak but are unlikely to be sufficient to eliminate community transmission in an acceptable amount of time.

- Stage 4 restrictions are likely to be sufficient to contain a Delta variant outbreak if implemented at a 7-day average of 30 cases per day

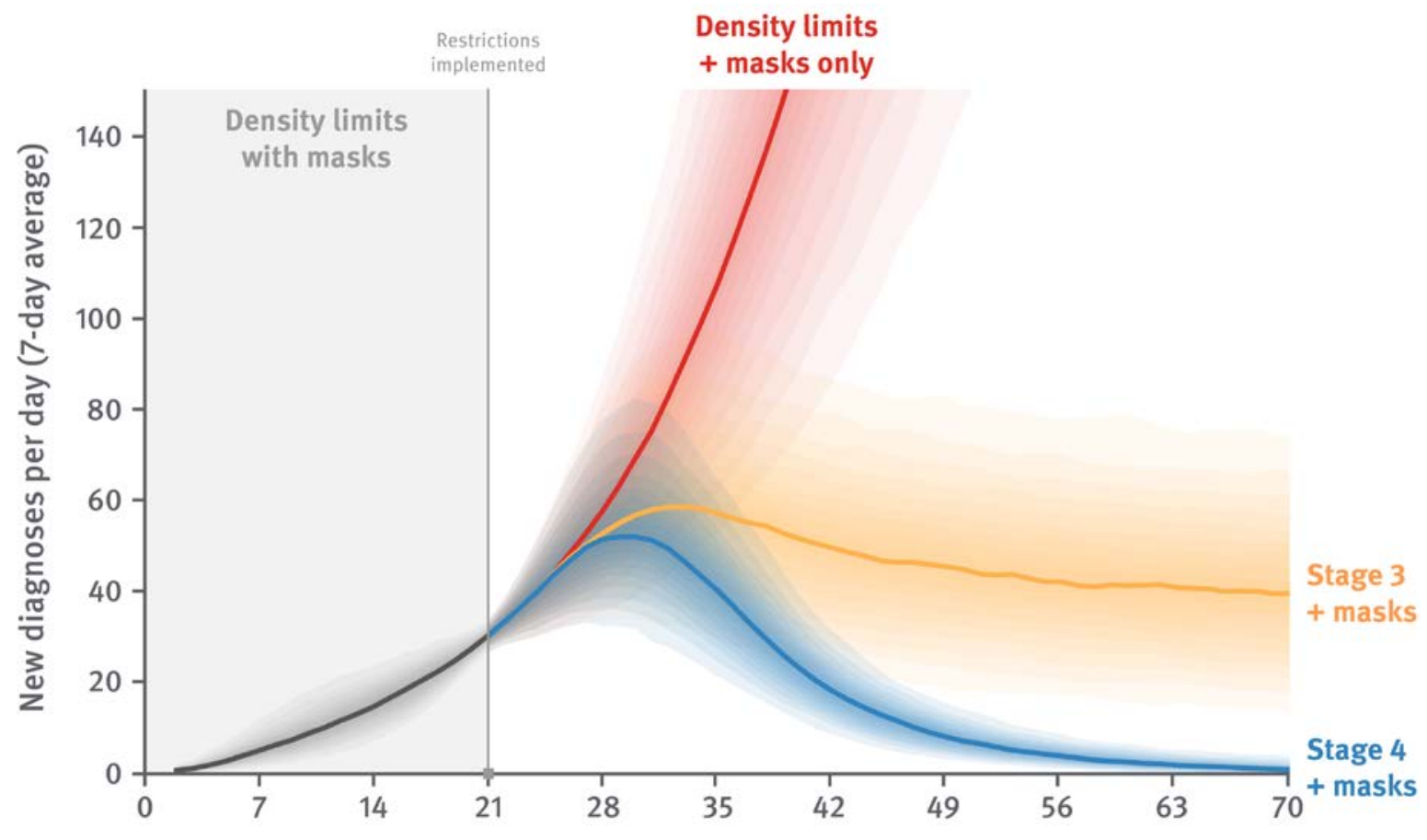

Days after first COVID-19 diagnosis

A key limitation in our analysis is that we did not explicitly model NSW, and the ways in which demographics, contact networks, and testing/contact tracing may differ from Victoria. Our model is spatially homogeneous, but the NSW outbreak was relatively geographically clustered, therefore our results are interpreted as applying to the affected parts of Sydney at the time, rather than the city as a whole. Finally, we did not explicitly model the restrictions imposed in NSW, because the impact of their restrictions was yet to be observed. While we can qualitatively assess the NSW restrictions relative to Victoria, some interventions (notably outdoor masks and curfews) are likely to have secondary effects on compliance and thus indirectly affect transmission, but data to estimate these secondary effects is not available, limiting the extent to which we can estimate the performance of policy responses that differ from Victoria's restrictions.

Implications for policymakers
- NSW's restrictions (as at 9 July 2021) were approximately equivalent to Victoria's Stage 3 restrictions, meaning that the approach being implemented was unlikely to return the epidemic to zero COVID in an acceptable amount of time.

- Increasing restrictions to the Stage 4 level used in Victoria in 2020 would likely be effective. 


\section{Case Study 2}

Model

Authors

Model

purpose

Model

Architecture

Model inputs

and starting

conditions

Results
The University of Melbourne

Melbourne School of Population and Global Health

Transport, Health and Urban Design Research Hub

University of New England, Faculty of Medicine and Health

This model was built to explore public health policy scenarios associated with COVID-19

in and outside Australia and their health and economic outcomes ${ }^{32-36}$. In this instance it

was tuned to estimate the likelihood of the State of New South Wales (NSW), Australia

returning to fourteen-day average of 5 confirmed cases per day under various restriction

policy scenarios of differing stringencies.

This is an agent-based model (ABM) that simulates a base population of 2500 agents, but can scale up to the population of NSW ( 8 million) or beyond.

The model accounts for individual mobility patterns and the effect of movement restrictions across the population. It can incorporate demographics and household size distributions, essential worker classifications, infection and disease transmission patterns, vaccine uptake and efficacy, differences in SARS-CoV-2 variants, track \& trace and isolation efficacy, compliance with restrictions, fatigue over time, capacity to work from home, interstate and overseas quarantine incursions, mask wearing, and individual decision-making.

The model can implement top-down policies including staged restrictions and associated public health interventions across pre-defined stages (e.g., Stage 1 to 4 ) in a dynamic or on an ad-hoc basis in response to current epidemiological conditions. These may include a combination of movement and economic restrictions, face-masks, distancing and density requirements, working from home requirements, vaccination regimes, or tracking and tracing initiatives such as mobile phone applications.

The model was calibrated to reflect conditions in the state of New South Wales, Australia in mid-July, 2021.

Three different policy scenarios were modeled:

1. Stage 2: Minimal restrictions in place (density and gathering caps) corresponding to the situation early in the NSW Delta outbreak.

2. Stage 3: Approximating restrictions in place in NSW between 9 and 17 July, 2021 (limited movement restrictions, some retail closed, schools closed, work from home if possible, home visit restrictions, indoor masks)

3. Stage 4: Hard lockdown based on the announcements made by the NSW government on the 18 July (e.g., movement restrictions, all non-essential economic activity closed, work from home mandate).

The model was seeded with an initial set of confirmed cases approximating 100 per day.

Each red line in the panel of charts, below represents one of 10,000 individual runs of the model under each policy scenario. The charts capture the uncertainty of infection patterns and outcomes under each policy setting. The black line represents the median across all model runs. The dotted lines bound the 5 th to 95 th percentile, providing a $90 \%$ range of what may occur.

Results indicated that a Stage 4 lockdown implemented on Sunday July 18th could reduce the number of daily cases to 5 or less per day in approximately 5.8 weeks - with a range of 4.6 to 7.5 weeks.

Under stage 3 restrictions, it estimated reaching $<=5$ cases per day would have taken 8.3 weeks - with a range of 5 to 14 weeks. 
A stage 2 scenario would likely have taken 14 weeks to get cases down to <=5 per day, even allowing for increasing vaccine coverage, but the range of times is becomes very uncertain - between 6 weeks and 8 months.

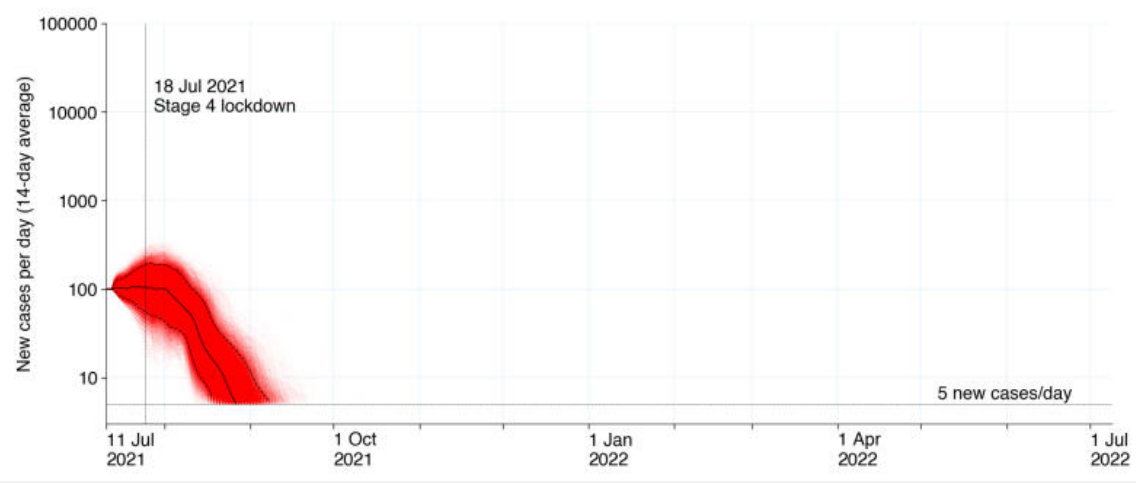

Implications for policymakers
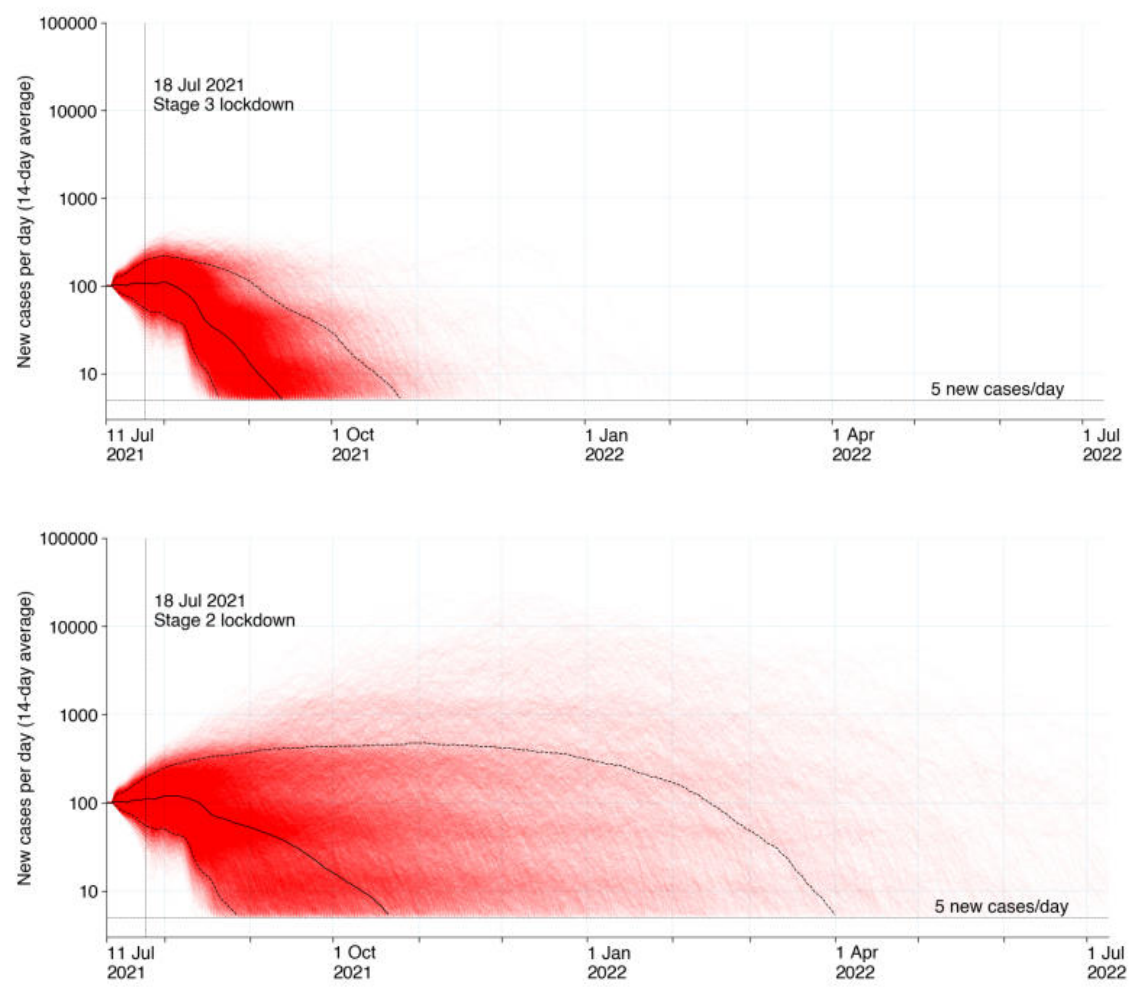

In early to mid-July, NSW had the opportunity to implement public health restrictions that would curtail their second major wave of COVID-19 infections, reducing time under stay-at-home orders to within approximately 6 weeks.

As of early August, 2021, NSW Government is maintaining restrictions that are roughly equivalent to Stage-3 levels, above. Implications are that without an effective tightening of restrictions that match stage-4 levels, NSW would be fortunate to return to less than 5 cases per day prior to October.*

*Since this model was released, it has been updated to reflect emerging information on the shorter generation and incubation times of the Delta variant ${ }^{37}$, as well as evidence regarding compliance and spread across different demographic groups across Sydney. This has not altered the core messages or implications. 
Case Study 3

Model

Authors

Model

Purpose

Model

Architecture

Model inputs and starting conditions
Sheryl L. Chang ${ }^{1}$, Oliver M. Cliff ${ }^{1,2}$, Cameron Zachreson ${ }^{1,3}$, Mikhail Prokopenko ${ }^{1,4}$

${ }^{1}$ The University of Sydney, Centre for Complex Systems

${ }^{2}$ The University of Sydney, School of Physics

${ }^{3}$ The University of Melbourne, School of Computing and Information Systems

${ }^{4}$ The University of Sydney, Sydney Institute for Infectious Diseases

The study ${ }^{38}$ aimed to identify a feasible range of non-pharmaceutical interventions for controlling a continuing outbreak of the B.1.617.2 (Delta) variant of SARS-CoV-2 in Sydney, Australia. In a nowcasting mode, it aimed to quantify the current levels of compliance with the restrictions. In a counter-factual mode, it aimed to quantify duration of control measures between the incidence peak and the time when new daily cases reduce below 10 . The study was based on an agent-based model (ABM) for transmission and control of COVID-19, validated for previous pandemic waves and genomic surveillance in Australia ${ }^{39-41}$. It was implemented within a large-scale software simulator (AMTraC-19) comprising approximately 23.4 million agents. Attributes of these stochastically generated agents matched key demographic and commuting patterns derived from the Australian Census and the Australian Curriculum, Assessment and Reporting Authority datasets ${ }^{42}$. AMTraC-19 simulated, in discrete time, interactions across several social mixing contexts: households, household clusters (neighbourhoods), communities, and workplace/study environments.

An initial distribution of infection was seeded by imported cases generated in proximity to the Sydney international airport. Each simulated outbreak was traced over time, updating in 12-hour cycles ("day-time" and "night-time"). Case-targeted non-pharmaceutical intervention (NPI) measures, such as case isolation and home quarantine, are in effect for the duration of each scenario. A pre-defined threshold ( 20 detected total cases) triggered border closures, limiting scenarios to local transmissions only. Another threshold triggered lockdown NPI measures, defined through (i) macro-distancing: modifying the population fraction complying with social distancing and school closures, and (ii) micro-distancing: modifying the interaction strengths for each complying agent, see Table 1 . The study compared two lockdown triggers: 100 and 400 detected total cases.

Table 1. Distancing parameters and interaction strengths for lockdown scenarios.

\begin{tabular}{|l|c|c|c|c|}
\hline \multirow{2}{*}{ NPI } & \multirow{2}{*}{ Compliance } & \multicolumn{3}{|c|}{ Interaction strength } \\
\cline { 3 - 5 } & & Household & Community & Workplace \\
\hline Case isolation & 0.7 & 1.0 & 0.1 & 0.1 \\
\hline Home quarantine & 0.5 & 2.0 & 0.1 & 0.1 \\
\hline School (students) & 1.0 & 1.0 & 0.1 & 0.0 \\
\hline School (parents) & 0.5 & 1.0 & 0.1 & 0.0 \\
\hline Social distancing & $0.7,0.8,0.9$ & 1.0 & 0.1 & 0.1 \\
\hline
\end{tabular}

The model was calibrated for B.1.617.2 (Delta) variant, using latest available information ${ }^{43,44}$, see Table 2, detailing the reproductive number $R_{0}$, the generation period $T_{\text {gen, }}$, the incubation period $\mathrm{T}_{\text {inc, }}$ and the post-incubation infectious period $\mathrm{T}_{\text {rec }}$.

Table 2. Parameters for the natural history of disease.

\begin{tabular}{|l|c|c|c|c|}
\hline & $\mathrm{R}_{0}$ & $\mathrm{~T}_{\text {gen }}$ (days) & $\mathrm{T}_{\text {inc }}$ (days) & $\mathrm{T}_{\text {rec }}$ (days) \\
\hline Mean & 5.97 & 6.88 & 4.4 & 10 \\
\hline $95 \% \mathrm{Cl}$ & $5.93-6.00$ & $6.81-6.94$ & $3.9-5.0^{7}$ & range: 7-14 (uniform) \\
\hline
\end{tabular}


The fraction of symptomatic cases was set 0.67 for adults, and 0.134 for children. Asymptomatic infectivity was modified by factor of 0.5 of the symptomatic or presymptomatic infectivity (the latter two were assumed to be equal). Detection probabilities were differentiated between symptomatic ( 0.23 per day) and asymptomatic/presymptomatic (0.01 per day).

Finally, the study compared two vaccination scenarios: either a limited pre-pandemic phase, or a progressive rollout achieving only partial mass-vaccination coverage within the projected lockdown duration. The components of the vaccine efficacies were set to match the latest available estimates ${ }^{41,45,46}$.

\begin{tabular}{l|l} 
Results & The level of social distancing currently attained in Sydney was found to be inadequate for
\end{tabular} the outbreak control. The closest match to the actual incidence data for the period up to 13 July 2021 was produced by a moderate macro-level of social distancing compliance, $S D=$ 0.4 , as well as moderately reduced interaction strengths within community. A higher compliance level, $S D=0.6$, provided the best match for the actual incidence dynamics during the period of more strict lockdown restrictions (16-25 July). The estimated levels of compliance with social distancing were supported by real-world Google mobility reports ${ }^{47}$. Assuming that a fraction of the population (up to $30 \%$ ) is engaged in essential services, the study suggested that the remaining fraction of the population (10-20\%) have not been consistently complying with the lockdown restrictions.

A counter-factual analysis considered higher levels of social distancing compliance: $70 \%$, $80 \%$ and $90 \%$, under both vaccination approaches, see Table 3 . The study found that, for $80-$ $90 \%$ SD compliance, the post-peak duration of lockdown is shorter for the scenario with an earlier lockdown (trigger: 100) and a pre-pandemic vaccination phase (the vaccination uptake set at 6\%, matching the levels attained in Australia around mid-June 2021). Conversely, $70 \%$ SD compliance performed better for the scenario with a delayed lockdown (trigger: 400 ) and a progressive vaccination rollout (projected to reach $40 \%$ by midSeptember 2021). This comparative analysis suggested that the benefits of a partial rollout, progressing over a short timeframe (approximately two months), are counter-balanced by a delayed lockdown imposition. Thus, the continuing vaccination rollout will make a stronger impact only at a longer timeframe (beyond two months).

Table 3. Projected duration of control measures after the incidence peak.

\begin{tabular}{|l|c|c|c|c|c|}
\hline \multirow{2}{*}{$\begin{array}{l}\text { Vaccination } \\
\text { scenario }\end{array}$} & Vaccination & \multirow{2}{*}{$\begin{array}{c}\text { Lockdown } \\
\text { uptake }\end{array}$} & \multicolumn{3}{|c|}{$\begin{array}{c}\text { Days after the peak until new } \\
\text { trigger } \\
\text { cases reduce below 10 per day }\end{array}$} \\
\cline { 4 - 6 } & & (total cases) & $70 \%$ SD & $80 \%$ SD & $90 \%$ SD \\
\hline Pre-pandemic & $6 \%$ & 100 & 55 & 28 & 17 \\
\hline Progressive & $\rightarrow 40 \%$ & 400 & 45 & 33 & 25 \\
\hline
\end{tabular}

Implications for policymakers
A stronger compliance and a careful rescoping of essential services (appropriately complemented by adequate financial support for the affected businesses and employees) are both needed to suppress the continuing Sydney outbreak of the Delta variant within a reasonable timeframe. Given low levels of the acquired immunity, a suppression can be shown only for the higher compliance with social distancing ( $S D \geq 0.7)$, as well as strongly reduced interactions outside of households. 


\section{References}

1. De Marchi S, Page SE. Agent-based models. Annual Review of political science 2014; 17: 1-20.

2. Biggs R, de Vos A, Preiser R, Clements H, Maciejewski K, Schlüter M. The Routledge Handbook of Research Methods for Social-Ecological Systems. Taylor \& Francis; 2021.

3. Epstein JM, Cummings DA, Chakravarty S, Singha RM, Burke DS. Toward a containment strategy for smallpox bioterror: An individual-based computational approach: Brookings Institution Press; 2004.

4. Thompson J, McClure R, de Silva A. A complex systems approach for understanding the effect of policy and management interventions on health system performance. Social-Behavioral Modeling for Complex Systems 2019: 809-31.

5. Kuhn T. The structure of scientific revolutions: Princeton University Press; 1962.

6. Trochim WM, Cabrera DA, Milstein B, Gallagher RS, Leischow SJ. Practical challenges of systems thinking and modeling in public health. American journal of public health 2006; 96(3): 538-46.

7. Waldherr A, Wijermans N. Communicating Social Simulation Models to Sceptical Minds. Jasss-J Artif Soc S 2013; 16(4): 13.

8. Tracy M, Cerdá M, Keyes KM. Agent-Based Modeling in Public Health: Current Applications and Future Directions. Annual review of public health 2018; 39(1): 77-94.

9. Spooner F, Abrams JF, Morrissey K, et al. A dynamic microsimulation model for epidemics. Social Science \& Medicine 2021; 291: 114461.

10. Blakely T, Thompson J, Bablani L, et al. Association of Simulated COVID-19 Policy Responses for Social Restrictions and Lockdowns With Health-Adjusted Life-Years and Costs in Victoria, Australia. JAMA Health Forum 2021; 2(7): e211749-e.

11. Casti JL. X-events: The collapse of everything: Harper Collins; 2012.

12. Walport M, Calder M, Craig C, et al. Computational modelling: technological futures. 2018.

13. Epstein JM. Why model? Journal of Artificial Societies and Social Simulation 2008; 11(4): 12.

14. Squazzoni F, Polhill JG, Edmonds B, et al. Computational Models That Matter During a Global Pandemic Outbreak: A Call to Action. Journal of Artificial Societies and Social Simulation 2020; 23(2): 10.

15. Monks T, Currie CSM, Onggo BS, Robinson S, Kunc M, Taylor SJE. Strengthening the reporting of empirical simulation studies: Introducing the STRESS guidelines. J Simul 2019; 13(1): 55-67.

16. van Dam KH, Nikolic I, Lukszo Z. Agent-based modelling of socio-technical systems: Springer Science \& Business Media; 2012.

17. Weiss $\mathrm{CH}$. The many meanings of research utilization. Public administration review 1979; 39(5): 426-31.

18. Walker WE, Lempert RJ, Kwakkel JH. Deep uncertainty. Delft University of Technology 2012; 1: 2.

19. Wilenius M, Casti J. Seizing the X-events. The sixth K-wave and the shocks that may upend it.

Technol Forecast Soc 2015; 94: 335-49.

20. McKinsey \& Co. When nothing is normal: Managing in extreme uncertainty, 2020.

21. Flannery T. The megafires and pandemic expose the lies that frustrate action on climate change. Australia: The Guardian, 2020.

22. Kalra N, Hallegatte $\mathrm{S}$, Lempert R, et al. Agreeing on robust decisions: new processes for decision making under deep uncertainty: The World Bank; 2014.

23. Ryan M. If you need to be right before you move, you will never win. Perfection is the enemy of the good when it comes to emergency management. Geneva, Switzerland: World Health Organization; 2020.

24. Sterman JD. Learning from evidence in a complex world. Am J Public Health 2006; 96(3): 505-14. 25. Orton L, Lloyd-Williams F, Taylor-Robinson D, O'Flaherty M, Capewell S. The Use of Research Evidence in Public Health Decision Making Processes: Systematic Review. PloS one 2011; 6(7): e21704.

26. Tangney P. Between conflation and denial - the politics of climate expertise in Australia. Australian Journal of Political Science 2019; 54(1): 131-49. 
27. Clarke EM, Wing JM. Formal methods: State of the art and future directions. ACM Computing Surveys (CSUR) 1996; 28(4): 626-43.

28. Clarke EM, Henzinger TA, Veith H. Introduction to model checking. Handbook of Model Checking: Springer; 2018: 1-26.

29. Cetinkaya O, Cetinkaya D. Verification and validation issues in electronic voting. Electronic journal of e-government 2007; 5(2).

30. Kingdon JW. How do issues get on public policy agendas. Sociology and the public agenda 1993; 8(1): 40-53.

31. Murray CJ, Frenk J. A framework for assessing the performance of health systems. Bull World Health Organ 2000; 78(6): 717-31.

32. Thompson J, Stevenson M, Blakely T, McClure R. Modelling Victoria's escape from COVID-19. Pursuit Magazine. 2020 September 8th, 2020.

33. Blakely T, Thompson J, Bablani L, et al. Determining the optimal COVID-19 policy response using agent-based modelling linked to health and cost modelling: Case study for Victoria, Australia. medRxiv 2021: 2021.01.11.21249630.

34. Blakely T, Thompson J, Carvalho N, Bablani L, Wilson N, Stevenson M. The probability of the 6week lockdown in Victoria (commencing 9 July 2020) achieving elimination of community transmission of SARS-CoV-2. Medical Journal of Australia 2020; 213(8): 349-51 e1.

35. State Government of Victoria. Emerging from lockdown: Evidence, modelling, outputs and assumptions. In: Services DoHaH, editor. Melbourne, Victoria: State Government of Victoria; 2020.

36. State Government of Victoria. Emerging from lockdown - model. In: Services DoHaH, editor. Melbourne, Victoria: State Government of Victoria; 2020.

37. Zhang M, Xiao J, Deng A, et al. Transmission Dynamics of an Outbreak of the COVID-19 Delta Variant B. 1.617. 2-Guangdong Province, China, May-June 2021. China CDC Wkly 2021; 3: 584-6.

38. Chang S, Cliff OM, Prokopenko M. Nowcasting transmission and suppression of the Delta variant of SARS-CoV-2 in Australia. ArXiv 2021; abs/2107.06617.

39. Chang SL, Harding N, Zachreson C, Cliff OM, Prokopenko M. Modelling transmission and control of the COVID-19 pandemic in Australia. Nature Communications 2020; 11(1): 5710.

40. Rockett RJ, Arnott A, Lam C, et al. Revealing COVID-19 transmission in Australia by SARS-CoV-2 genome sequencing and agent-based modeling. Nature Medicine 2020; 26(9): 1398-404.

41. Zachreson C, Chang SL, Cliff OM, Prokopenko M. How will mass-vaccination change COVID-19 lockdown requirements in Australia? The Lancet Regional Health - Western Pacific 2021; 14(1).

42. Fair KM, Zachreson C, Prokopenko M. Creating a surrogate commuter network from Australian Bureau of Statistics census data. Scientific Data 2019; 6(1): 150.

43. Campbell F, Archer B, Laurenson-Schafer $\mathrm{H}$, et al. Increased transmissibility and global spread of SARS-CoV-2 variants of concern as at June 2021. EUROSURVEILLANCE 2021; 26(24): 2100509.

44. Zhang M, Xiao J, Deng A, et al. Transmission Dynamics of an Outbreak of the COVID-19 Delta Variant B.1.617.2 - Guangdong Province, China, May - June 2021. China CDC Weekly 2021; 3: 584-6.

45. Bernal JL, Andrews N, Gower C, et al. Effectiveness of COVID-19 vaccines against the B.1.617.2 variant. medRxiv 2021: 2021.05.22.21257658.

46. Harris RJ, Hall JA, Zaidi A, Andrews NJ, Dunbar JK, Dabrera G. Effect of Vaccination on Household Transmission of SARS-CoV-2 in England. New England Journal of Medicine 2021.

47. Google. Community Mobility Reports. 2021. https://www.google.com/covid19/mobility/ (accessed 25th July 2021. 